\title{
Evidence-based Clinical Practice in Rehabilitation
}

This issue of Brain Impairment includes topic reviews on prospective
memory (Shum, Fleming, \& Neulinger) and aphasia therapy (Douglas,
Brown, \& Barry). While Shum and colleagues provide a narrative review on
prospective memory, the aphasia therapy paper presents an overview of sys-
tematic reviews in the tradition of evidence-based clinical practice (EBCP).

$\mathrm{EBCP}$ is not new but it has recently been taken up and applied to the clinical practice of medicine (see Sackett et al., 1997; 2000). EBCP rests on the premise that there are varying degrees of the quality of evidence for particular procedures or interventions. In other words, some evidence is stronger than other evidence. The drive is to train clinicians and students to be knowledgeable and critical about the various levels of evidence so that this will inform their clinical practice. As applied to rehabilitation provided by allied health clinicians, for example, in 1999 Carney and colleagues published a systematic review on the efficacy of cognitive rehabilitation after traumatic brain injury. They identified 32 reports, comprising 11 randomised controlled trials, four comparative studies and 17 observational studies. A number of studies provided support for the efficacy of cognitive rehabilitation, and others did not find any treatment effects. So, is or is not cognitive rehabilitation effective? Examining the methodological quality of the studies in terms of the level of evidence provides the framework for decision-making. The evidence provided by flawed studies or those with weak designs should be less influential for the clinician's evaluation as to whether cognitive rehabilitation works or not.

Levels of evidence have traditionally been defined as follows: The firmest evidence (Class I) is provided by well-designed randomised controlled trials (RCT). Class IIa refers to RCT with design flaws, multicentre or population-based longitudinal (cohort) studies. Class IIb level of evidence comes from nonrandomised controlled trials, case-control studies and well designed case series. Class III level of evidence is provided by case reports, uncontrolled case series and expert or consensus opinion. The National Health and Medical Research Council (1999) guidelines recommend the addition of another class of evidence. Systematic reviews are now regarded as providing the strongest level of evidence. A systematic review summarises the results from all the studies in a certain area. They are much more than meta-analyses because they adopt a comprehensive strategy for searching primary studies, as well as explicating inclusion and exclusion criteria of studies in the review.

In rehabilitation practice we need to reflect upon the issue of the extent to which our clinical work is based upon the best evidence available, and how much it is based on the lowest level of evidence, consensus opinion. Cicerone, Dahlberg, Kalmar, et al. (2000) used evidence-based practice recommendations to bridge the conceptual gap between levels of evidence and clinical practice nicely. Evidence-based practice recommendations are practice guidelines that have been systematically developed to assist the 
decision-making of clinicians and patients about appropriate health care for specific clinical circumstances. The evidence upon which recommendations are based is crucial to their validity. Consequently, practice recommendations are frequently graded according to the quality of the supporting evidence upon which they are based. Cicerone and colleagues proposed three levels of recommendations defined by the strength of associated evidence. Practice Standards required the strongest level of evidence and were based on at least one, well-designed Class I study with an adequate sample, or overwhelming Class II evidence, that directly addressed the effectiveness of the treatment in question. Practice Guidelines required fair evidence to support the recommendation and were based on well-designed Class II studies with adequate samples. Practice Options had the lowest level of evidence and unclear clinical certainty. They were based on Class II or Class III studies and require additional grounds to support a recommendation as to whether the treatment be specifically considered.

One problem for clinicans in rehabilitation practice, however, is the dearth of systematic reviews and Class I studies; another problem is the difficulty of accessing information effectively and efficiently. The former need can only be addressed by further research using strong methodological designs; the latter by developing specialised databases. It is encouraging to see the enthusiasm with which allied health disciplines are building foundations for EBCP in their own disciplines, and Australia is in the forefront of these developments. In 2000, Brain Impairment published a paper by Moseley, Sherrington, Herbert and Maher, describing PEDro, the Physiotherapy Evidence Database. This free internet-based resource of systematic reviews and RCT can be accessed through their web site: http://ptwww.cchs.usyd. edu.au/pedro/. Recently, the Motor Accidents Authority of NSW has funded two projects to develop similar databases, one in occupational therapy (McKenna, Bennett, Strong, Tooth, \& McCluskey), entitled OT-SEEker (Occupational Therapy-Systematic Evaluation of Evidence) and another for psychological therapies, including language, after acquired brain injury (Tate, Perdices, McDonald, Togher, \& Moseley), entitled PsychBITE ${ }^{\mathrm{TM}}$ (Psychological database for Brain Injury Treatment Efficacy). Brain Impairment is keen to publish work that supports evidence-based clinical practice.

In this issue, we also have the sad task of publishing two obituaries of our colleagues and friends. Hamish Godfrey died earlier this year and Dr Robert Knight, Head of the Department of Psychology at Otago University, has written an obituary. Hamish was a member of the Editorial Board of Brain Impairment. He was extremely positive and encouraging about the launch of the journal, and worked hard on providing reviews for early papers. We will miss his contribution and involvement. Dr Dorothy Gronwall also died late last year. Dorothy was legendary in the field of neuropsychology, establishing an international reputation in the mid-1970's. Dr Jenni Ogden, Associate Professor in the Department of Psychology at Auckland University, has written an obituary. The Editors and Editorial Board of Brain Impairment extend our condolences to the families, friends and colleagues of Hamish and Dorothy.

\section{References}

Carney N., Chestnut, R.M., Maynard, H., Mann, N.C., Patterson, P., \& Helfand, M. (1999). Effect of cognitive rehabilitation on outcomes for persons with traumatic 
brain injury: A systematic review. Journal of Head Trauma Rehabilitation, 14(3), 277-307.

Cicerone, K., Dahlberg, C., Kalmar, K., Langenbahn, D., Malec, J., Bergquist, T., et al. (2000). Evidence-based cognitive rehabilitation: Recommendations for clinical practice. Archives of Physical Medicine and Rehabilitation, 81, 1596-1615.

Moseley, A., Sherrington, C., Herbert, R., \& Maher, C. (2000). The extent and quality of evidence in neurological physiotherapy: An analysis of the Physiotherapy Evidence Database (PEDro). Brain Impairment, 1, 130-140.

National Health and Medical Research Council. (1999). A guide to the development, implementation and evaluation of clinical practice guidelines. Canberra: Commonwealth of Australia.

Sackett, D.L., Richardson, W.S., Rosenberg, W., \& Haynes, R.B. (1997). Evidence-based medicine: How to practice and teach EBM. New York: Churchill Livingstone.

Sackett, D.L., Straus, S.E., Richardson, W.S., Rosenberg, W., \& Haynes, R.B. (2000). Evidence-based medicine: How to practice and teach EBM (2nd ed.). New York: Churchill Livingstone.

\section{Robyn Tate, $\mathrm{PhD}$}

Rehabilitation Studies Unit, Department of Medicine, University of Sydney and Royal Rehabilitation Centre Sydney

\section{Jacinta Douglas, $\mathrm{PhD}$}

School of Human Communication Sciences, Faculty of Health Sciences, La Trobe University 